\title{
Design of the Antagonistic Simulation Training Platform Based on Jini
}

\author{
Hongmei Zhang ${ }^{1, a}$, Wenqian Zhang ${ }^{2, b^{*}}$ and Peng $X^{1, c}$ \\ ${ }^{1}$ Science College, Air Force Engineering University, Xi'an 710051, China; \\ ${ }^{2}$ Equipment Management and Safety Engineering College , Air Force Engineering University, Xi'an \\ 710051, China. \\ azhm_plum@163.com, b664599317@qq.com, 'xpf68@163.com
}

Keywords: Jini; network-configuration; service; plug and play; simulation training grid

\begin{abstract}
With the development of network-centric warfare theory, a variety of networking equipment has become a serious problem. In the process of networking, because of different equipment, different manufacturers and models of the same type equipment, the network is needed to standardize the process of data formats and interfaces, which bring a lot of problems to the network equipments. To solve these problems, antagonistic emulation training platform is designed based on Jini. Jini uses of plug-and-play technology, as well as self-organized, self-healing features to support a wide range of combat training and seamless interconnection between equipment, to construct "system-wide" integrated simulation training environment.
\end{abstract}

\section{Introduction}

Eyes on the future integration of joint operations command and the need for training, exploration and building of my Air Force features tactical simulation training platforms is an important task. Tactical simulation training platform is designed to integrate existing sensor networks ${ }^{[1-2]}$, command control networks and a variety of simulation training system to form a grid-based confrontational simulation training platform. The significance of the grid of simulation training platform lies in:

(1) conducive to the formation of "system-wide" integrated simulation training environment;

(2) conducive to full use of the existing training resources and reduce repeated investment;

(3) improve training efficiency.

Simulation training and combat grid have the same architecture, simulated training equipment can be connected to grid during peacetime training, while the actual equipment is connected in wartime .So we can use the closer way of actual combat training to improve day-to-day training efficiency.

Simulation training equipment has many forms, such as physical simulation, half-physical simulation, as well as the all-digital simulation. It will cause enormous difficulties to unify their interface and data format during the construction of network. In order to avoid such problems, and make different models, different types of simulation training equipment or reality equipment connect to the training network, in this article, the author advance a way that using Jini technology to implement network "plug and play" solution, and design the form of network.

\section{Jini Technology}

In January 1999, Sun released a new distributed system technology ---Jini ${ }^{[3-5]}$. It is a distributed system based on the idea of federating groups of users and the resources required by those users. The focus of the system is to make the network a more dynamic entity that better reflects the dynamic nature of the workgroup by enabling the ability to add and delete services flexibly.

A Jini system uses the network as a foundation to enable service discovery and service execution. Traditional systems attempt to mask the appearance of the network; in contrast, Jini uses the dynamic, flexible nature of the network to form communities and register, discover, and invoke services. Unlike traditional systems, Jini is founded on the assumptions that networks are unreliable, change frequently, and should not require substantial maintenance by an administrator. 


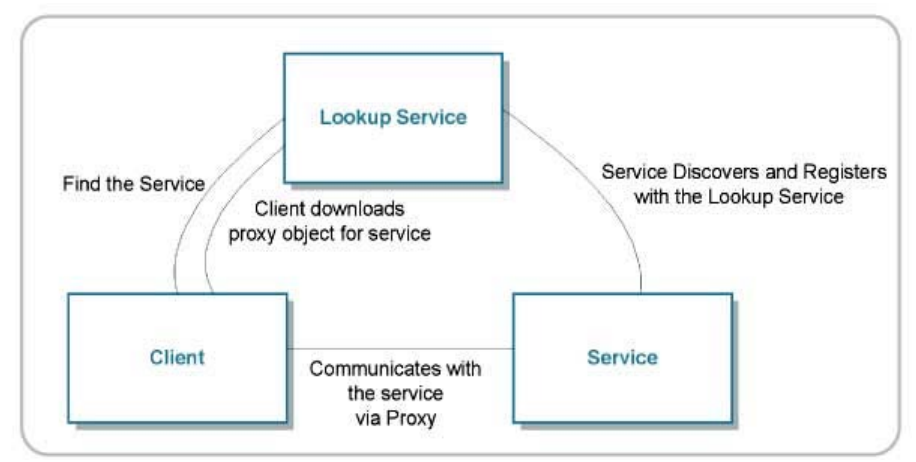

Figure1: Jini architecture

The Jini architecture (see Figure 1) is comprised of the following elements:

(1) Jini Service

A Jini service is an entity that can be a hardware device or a software component that provides functions like printing a report, looking up a stock quote, or executing an algorithm. Applications and other Jini services can use a Java interface and a Java proxy class to access a Jini service. The Java interface provides the definition of the methods that are available from the Jini service. In most cases, the underlying system that allows Jini services to communicate is RMI. A Jini service may also use protocols such as SOAP, XML/HTTP, or CORBA.

(2) Lookup Service

The lookup service, which is a Jini service, keeps track of the Jini services and provides proxies to communicate with the services. Sun provides a lookup service called Reggie (used for developing this example) as part of the Jini development kit.

(3) Jini Client

The Jini client is any software that requests the proxy from the lookup service in order to invoke the Jini service.

Jini can make a variety of digital devices work together at a temporary equipment called the "Alliance Services" (or known as the "community") without configuration, installation, or manual intervention. Through the use of leased machinery, equipment in any league, regardless of their size can be self-managed and jointly form a service network, each member of the league can provide resources or services, while at the same time can obtain their own needed resources and services from the other members.

Given the advantages and mechanisms of Jini technology, we can use it to construct confrontational simulation training platform.

\section{Design of confrontational simulation training platform}

\subsection{Ways of equipment access to Jini network}

Because of the different levels of intelligence, equipment can be divided to different categories. According to different situations, the ways of its access to network can be roughly divided into three types: implementation of the equipment based on the complete virtual machine v based on the embedded virtual machine 、 based on the sharing virtual machine.

(1)The equipment based on the complete virtual machine.

This generally refers to the use of general (such as computers) simulation training equipment. Usually ,this type of device has network connectivity and strong computing storage functions, being able to run a complete virtual environment Java2, achieving all the features of Jini system, such as a variety of protocol, RMI, lease update feature, dynamic code for mobile and distributed security mechanism, and so on.

(2)The equipment based on embedded virtual machine.

With the release of a wide variety of embedded Java virtual machine and operating system, some equipment have networking functionality. Compared to general-purpose equipment, such equipment usually has the following characteristics: embedded JVM is only a subset of a complete Java platform, which can be able to implement part of the core calculation functions of Jini. These devices only join 
or leave the Jini Commonwealth intermittently; these devices have smaller computing power and narrower bandwidth communications.

(3)The equipment based on sharing virtual machine.

Such equipment can be divided into two categories: one is that has little or no computing and storage capabilities, so it does not have the ability to run virtual machine equipment; the other is the traditional non-Jini-enabled devices. Both devices must function with other strong devices for sharing a virtual machine to join the Jini network, and register their own services or use of other registration service. The sharing approach has two kinds: local sharing and network sharing. Local sharing is that more than one devices connect to a local master device through the physical interface. At this point, the main equipment acts as other equipment's Jini agent, which will register the services provided by these equipment, and deal with the communication between them and the Jini community. Network sharing is similar with local sharing: the main equipment is still as other equipment's Jini agent and is responsible for various communications between these equipment and Jini communities, but interactive connection and communications are done through the network based on proprietary protocol or other standard communication protocols.

During the actual process of network construction, the majority of cases, we make use of Jini technology for the control of the equipment without JVM.

\subsection{Design of grid structure $\mathrm{e}^{[6-8]}$}

Simulation training system adopts multi-layer form, each layer of the network constitutes a flat grid and provide services for the higher layer.Its structure diagram shown in Figure2:

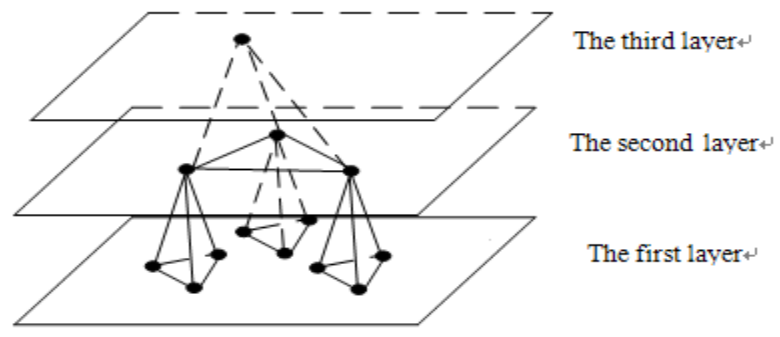

Figure2: The multi-layer structure of simulation training system grid

The bottom of the simulation training system grid is made up of a variety of simulated training equipment and simulation training system, as shown in Figure 3, including the simulation flight node, the missile launch control node, communication node, navigation node, and so on. Each node group in accordance with the Jini system. The main class of this layer is to complete the simulation training at equipment level, each hardware and software of the training equipment can be used as a Jini services which would be released. At the same level, all the inter-node information resources can be shared, and the same type of nodes can cooperate with each other in order to complete a mission, different types of nodes can be in accordance with each other to provide services required.

We can make use of Jini mechanisms to implement joining or leaving fighting. First, all the services must register at the lookup service and apply for the lease time. Secondly, simulation node participating in combat constitutes a Jini community, all the nodes search service at lookup service as a client and download service agent to a local machine, then each node can communicate with each other through the agent. The node destroyed will leave Jini community automatically as lease expiration. Each node no longer updated lease after training, and the information of lookup services would be deleted. Finally, the entire grid return its original state . 


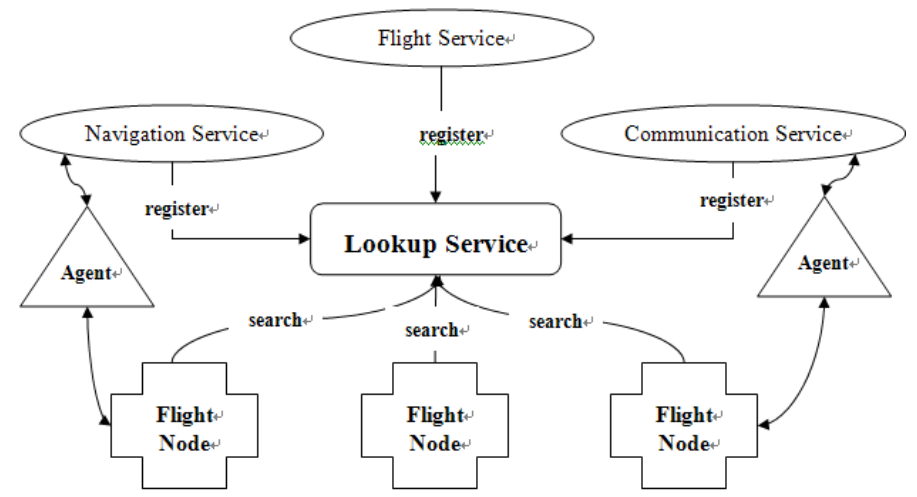

Figure 3:The underlying structure of simulation training system grid

The second layer is to complete simulation training of the primary commanders, which requires the cooperation of the first layer. The nodes of the first layer provides coordinates, posture, route and other information to the second layer, where these messages can be integrated into the real-time information of the current battlefield situation. Then, commanders can make decisions in accordance with the battlefield situation, and transfer decision-making information to the first layer to complete the operational command simulation training.

The third layer, whose next layer may include coordination among the various branches of the armed forces, is aimed at the command training of senior commanders. From the organizational point of view, it may need more than one Jini communities.

\subsection{Example of network construction}

We take navigation, radar and satellite network construction as an example to show how to make full use of Jini to structure integration simulation training grid, assuming that the relationship setting between the forces participating in the training shown in Figure 4:

Decision-making is at the top tier, which makes decision based on info from radar information fusion center and satellite information fusion center.Then,it releases combat command to Air Force troops, which would complete mission under the cooperation of navigation troops. The data achieved from radar and satellite would be transferred to respective information fusion center for further treatment.

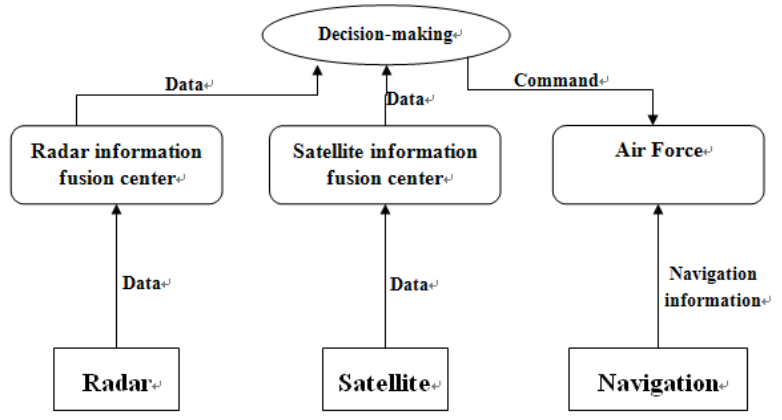

Figure 4 :The relationship setting between the forces participating in the training

According to mission needs and the relationship between combat units ,Jini network can be designed as the following forms:

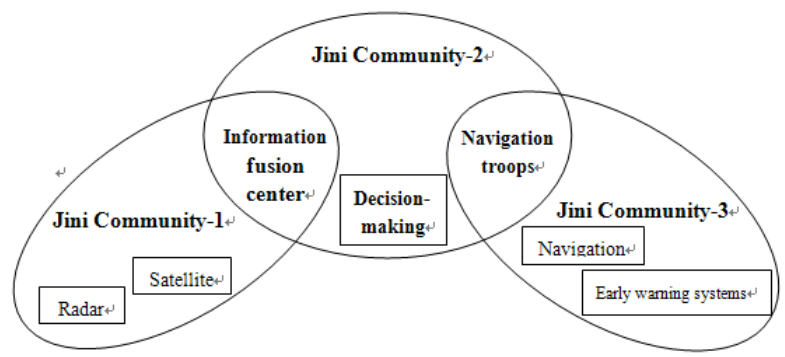

Figure5 :The form of Jini network

We divide the combat regional grid into three Jini communities. Community- 1 is composed of radar, satellite and information fusion center; Community-2 is composed of command 
decision-making and related services; Community-3 is composed of aviation simulation training system, navigation and early warning systems.

Radars register their data acquisition function as a service in Jini Community-1 through their agent. Information fusion center would search radar data service of interest in Jini Community-1 as one client. If the specified services are found, the radar will collect data and pass it to the fusion center, which complete data treatment. During this process, if a radar was destroyed, it would exit the community automatically as lease expiration. When there is "supplemental" radar connected to replace the destruction radar, the new one would be identified and called by other services as long as it register itself at Jini community.

At the same time, fusion center would play different roles in Jini. It will register its service in Community-2 that contains the decision-making tire. The fusion center will release the information that has been dealt with as a service in Community-2.Then the decision-making tire can search the services provided by fusion center from the lookup service of Community-2. The decision-making tire release mission to navigation troops and early warning systems that have been registered in Community-3, from which navigation troops and early warning systems obtain information. The joining and leaving of navigation and early warning systems services is similar with radar services.

\section{Conclusion}

The design of confrontational simulation training platform based on can make full use of Jini network system with plug-and-play and self-organization, self-healing features, and support seamless connection of a wide range of training or combat equipment, which is conducive to the formation of "system-wide" integrated simulation training environment. The use of Jini technology in current simulation training can improve training efficiency and reduce investment.

\section{References}

[1] Gents W. Grid computing, a vendor's vision [C]. In Cluster Computing and the Grid 2nd IEEE / ACM International Symposium CCGRD2002,2002,3:272-277.

[2] Jim Waldo. The Jini Architecture for Network-centric Computing. Communications of the ACM,42(7):76-82,July1999

[3] Jini technology community resource, http://www.jini.org/

[4] Liu Deyuan, Chen Huitang. Jini Applied Research [J]. Computer Application and Software, 2004,21 (8) :72-74.

[5] Shuai Chun-yan, Cheng Wen-ying,Lu Yang. Research of JINI Leasing Thought and Implementation Technology[J]. Journal of Kunming University of Science and Technology (Science and Technology),2003,28(6):61-64.

[6] Scott Rose, Kevin Bowers, Steve Quirolgico, Kevin Mills. Improving Failure Responsiveness in Jini Leasing. http://ieeexplore.ieee.org,2003.

[7] Joachim Posegga.Jini:Infrastruktur for dynamische Dienste in verteilten Systemen. Informatik Spektrum,1999,22(2):43-44.

[8] Nathalie Furmento, Anthony Mayer, Stephen McGough, Steven Newhouse,Tony Field and John Darlington, ICENI: Optimisation of Component Applications within a Grid Environment, submitted to Journal of Parallel Computing, February 2002. 